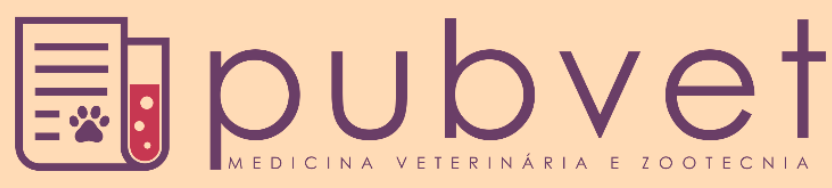

ISSN 1982-1263

https://doi.org/10.31533/pubvet.v13n7a369.1-7

\title{
Criptosporidiose bovina: aspectos clínicos, epidemiológicos e terapêuticos
}

\author{
Weslen Fabricio Pires Teixeira ${ }^{1 \bullet}$, Dielson da Silva Vieira ${ }^{2^{*}}$, Welber Daniel Zanetti Lopes ${ }^{1 \bullet}$, \\ Mariana Fachini Esperança ${ }^{30}$, Katia Denise Saraiva Bresciani ${ }^{2} \theta$ \\ ${ }^{I}$ Universidade Federal de Goiás, Goiânia. \\ ${ }^{2}$ Universidade Estadual de São Paulo (Unesp), Escola de Medicina Veterinária, Araçatuba. \\ ${ }^{3}$ Universidade Estadual de São Paulo (Unesp), Faculdade de Ciências, Bauru. \\ *Autor para correspondência, e-mail: dielson.vieira@ig.com.br
}

Resumo. A criptosporidiose bovina é causada principalmente pelas seguintes espécies do gênero Cryptosporidium: Cryptosporidium parvum, Cryptosporidium bovis, Cryptosporidium andersoni e Cryptosporidium rynae. O aparecimento de sinais clínicos em bovinos infectados está diretamente correlacionado a idade, estado imunitário do hospedeiro e da espécie do parasito responsável pela infecção. A diarreia é o sinal clínico mais evidente durante a criptosporidiose bovina, sendo normalmente observada em bezerros neonatos infectados por $C$. parvum. De todas as espécies do gênero Cryptosporidium que acomete bovinos, o $C$. parvum é a única que reconhecidamente apresenta potencial zoonótico. Os bovinos quando infectados por essa espécie, excretam grandes quantidades de oocistos do parasito no meio ambiente, fator muito importante na epidemiologia desta coccidiose. A ausência de drogas e vacinas eficazes contra a infecção por Cryptosporidium spp. em bovinos, apontam para a necessidade de medidas profiláticas voltadas ao manejo adequado dos animais. O objetivo desta revisão foi elucidar os aspectos clínicos, epidemiológicos e terapêuticos da criptosporidiose em bovinos.

Palavras-chave: coccídeo, Cryptosporidium, diarreia

\section{Bovine cryptosporidiosis: clinical, epidemiological and therapeutic aspects}

\begin{abstract}
Bovine cryptosporidiosis is caused mainly by the following species of the genus Cryptosporidium: Cryptosporidium parvum, Cryptosporidium bovis, Cryptosporidium andersoni and Cryptosporidium rynae. The appearance of clinical signs in infected cattle is directly correlated to the age, host immune status and the parasite species responsible for the infection. Diarrhea is the most evident clinical sign during bovine cryptosporidiosis, and it is usually observed in newborn calves infected with $C$. parvum. Of all species of the genus Cryptosporidium that affects cattle, $C$. parvum is the only one that is known to have zoonotic potential. Bovine animals, when infected by this species, excrete large amounts of oocysts of the parasite in the environment, a very important factor in the epidemiology of this coccidiosis. The absence of effective drugs and vaccines against Cryptosporidium spp. in cattle, point to the need for prophylactic measures aimed at the proper management of animals. The objective of this review was to elucidate the clinical, epidemiological and therapeutic aspects of cryptosporidiosis in cattle.
\end{abstract}

Key words: coccidia, Cryptosporidium, diarrhea

\section{Criptosporidiosis bovina: aspectos clínicos, epidemiológicos y terapéuticos}

Resumen. La criptosporidiosis bovina es causada principalmente por las siguientes especies del género Cryptosporidium parvum, Cryptosporidium bovis, Cryptosporidium 
andersoni y Cryptosporidium rynae. La aparición de signos clínicos en bovinos infectados es directamente correlacionada a la edad, estado inmunitario del huésped y de la especie del parásito responsable de la infección. La diarrea es el signo clínico más evidente durante la criptosporidiosis bovina, siendo normalmente observada en becerros neonatos infectados por C. parvum. De todas las especies del género Cryptosporidium que acomete bovinos, el C. parvum es la única que reconocidamente presenta potencial zoonótico. Los bovinos cuando se infectan por esa especie excretan grandes cantidades de oocistos del parásito en el medio ambiente, factor muy importante en la epidemiología de esta coccidiosis. La ausencia de drogas y vacunas eficaces contra la infección por Cryptosporidium spp. en bovinos, apuntan a la necesidad de medidas profilácticas dirigidas al manejo adecuado de los animales. El objetivo de esta revisión fue dilucidar los aspectos clínicos, epidemiológicos y terapéuticos de la criptosporidiosis en bovinos.

Palabras-clave: coccidio, Cryptosporidium, diarrea

\section{Introdução}

Desde o primeiro relato mundial da criptosporidiose bovina em 1971 (Panciera et al., 1971), estudos epidemiológicos vem sendo realizados obtendo diferentes resultados quanto a infecção por parasitos do gênero Cryptosporidium nestes animais (Fayer et al., 2008; Meireles et al., 2011; Taylan-Ozkan et al., 2016; Baroudi et al., 2017; Matsuura et al., 2017;).

O gênero Cryptosporidium é responsável pela infecção de bovinos de diferentes faixas etárias, podendo ocasionar a grandes perdas econômicas a pecuária (De Waele et al., 2011; Yap et al., 2016). Tais prejuízos geralmente são atribuídos aos tratamentos não efetivos dos animais infectados e ao aumento da mortalidade pós-natal provocada pela associação do parasito com agentes virais e/ou bacterianos (Feitosa et al., 2008). Além de perdas econômicas, a utilização indiscriminada de antibióticos no tratamento da criptosporidiose bovina, pode ainda, acelerar o mecanismo de resistência de enterobactérias aos princípios ativos utilizados (Feitosa et al., 2008).

Diferentes fatores como a idade, a imunocompetência e o sistema de criação animal, estão diretamente ligados à infecção por Cryptosporium spp. em bovinos (Feitosa et al., 2008; Garro et al., 2016; Wegayehu et al., 2016; Yap et al., 2016), sendo altas incidências do parasito correlacionas a condições precárias de manejo principalmente de bezerros neonatos e de vacas no período periparto, que podem eliminar quantidades expressivas de oocistos no ambiente (Feitosa et al., 2008).

O objetivo desta revisão foi apresentar um panorama geral sobre os aspectos clínicos, epidemiológicos e terapêuticos da criptosporidiose bovina.

\section{Epidemiologia e aspectos clínicos}

As espécies de Cryptosporidium mais prevalentes em bovinos são: o Cryptosporidium andersoni, o Cryptosporidium bovis, o Cryptosporidium ryanae e o Cryptosporidium parvum (Meireles et al., 2011; Muhid et al., 2011; Taylan-Ozkan et al., 2016; Wegayehu et al., 2016; Baroudi et al., 2017). Além da idade, do estado imunológico e do manejo, a ocorrência destes parasitos está diretamente correlacionada a linhagem animal, onde as maiores prevalências de Cryptosporidium spp. são diagnosticadas em animais taurinos (Wegayehu et al., 2016). Além destes fatores, a sintomatologia clínica da criptosporidiose bovina é dependente da espécie do parasito envolvida na infecção (Muhid et al., 2011). Em bezerros neonatos são geralmente diagnosticadas as maiores taxas de infecção por C. parvum, sendo nesta categoria animal evidenciadas maiores excreções de oocistos por grama de fezes (Feitosa et al., 2008; Meireles et al., 2011; Teixeira et al., 2011), fato oposto em animais adultos (pós-desmame), onde outras espécies do parasito (C. andersoni, C. bovis e C. ryanae) são mais prevalentes (Fayer, 2004).

O C. andersoni é um parasito de glândulas gástricas do abomaso de bezerros com idade superior a quatro semanas de vida e animais adultos imunocomprometidos (Fayer et al., 2008). Geralmente a infecção por esta espécie adota caráter crônico (sem o aparecimento de sinais clínicos), com excreção de oocistos que pode perdurar por até um ano. Apesar da infecção por $C$. andersoni em bovinos geralmente se apresentar assintomática, a diminuição na produção de ácidos gástricos do hospedeiro provocada pelo parasito pode comprometer a digestão de proteínas e consequentemente a produção dos 
animais (Morgan et al., 2000). Morfologicamente os oocistos de C. andersoni apresentam formato elipsoidal, com tamanho aproximado de 5,0 por $7,0 \mu \mathrm{m}$ e a presença em seu interior de quatro esporozoítos (Lindsay et al., 2000). Esta espécie, apesar de não ser considerada zoonótica, foi diagnosticada em amostras fecais humanas no Malawi e na Índia (Hussain et al., 2017; Morse et al., 2007).

Em bovinos de três meses a dois anos de idade, o C. bovis é a espécie mais prevalente (Fayer, 2004; Fayer et al., 2007). Com caráter assintomático, a infecção intestinal por este parasito proporciona a excreção de oocistos com aproximadamente 4,8 por $4,6 \mu \mathrm{m}$ e morfologicamente semelhantes aos de $C$. parvum e $C$. ryanae, sendo a diferenciação entre essas espécies, somente realizada por meio de técnicas moleculares (Fayer, 2004). A infecção por C. ryanae geralmente não proporciona o surgimento de sintomatologia clínica em bovinos, sendo sua maior taxa de ocorrência diagnosticada em animais com mais de três meses de idade (Fayer et al., 2007; Meireles et al., 2011). Morfologicamente, os oocistos desta espécie apresentam tamanho de 3,7 por 3,1 $\mu \mathrm{m}$ de diâmetro, sendo considerado o menor dentre todas as espécies de Cryptosporidium que infectam mamíferos (Fayer et al., 2008). C. parvum é a única espécie do parasito que acomete bovinos que apresentam comprovado potencial zoonótico (Dixon et al., 2011). A infecção por esta espécie em bovinos neonatos, geralmente é caracterizada pela replicação de formas parasitárias do coccídeo em células intestinais destes animais e consequentemente surgimento agudo de diarreia aquosa (Santín et al., 2007; Garro et al., 2016), com excreção de grandes quantidades de oocistos $\left(10^{7}\right.$ a $10^{10}$ por grama de fezes) com formato esférico e tamanho aproximado de 4,5 por 3,3 $\mu \mathrm{m}$ (Fayer et al., 2007; Santín et al., 2007). A ocorrência de diarreia na criptosporidiose bovina provocada por C. parvum geralmente aparece associada a infecções bacterianas, virais ou com outras causas parasitárias (Feitosa et al., 2008), podendo elevar consideravelmente os índices de mortalidade em bezerros infectados (Morse et al., 2007; Noordeen et al., 2012; Cacciò et al., 2013;).

\section{Diagnóstico}

As técnicas de microscopia ainda são consideradas as mais utilizadas na rotina laboratorial para o diagnóstico de oocistos de Cryptosporidium spp. em amostras fecais bovinas (Teixeira et al., 2011). As técnicas para detecção microscópica de oocistos de Cryptosporidum spp. mais utilizadas laboratorialmente no diagnóstico em bovinos são: a técnica de Kinyoun (Ma \& Soave, 1983), o ZiehlNeelsen modificado (Henriksen \& Pohlenz, 1981), a coloração por safranina e azul de metileno (Baxby et al., 1984), a coloração negativa por verde malaquita (Elliot et al., 1999), a microscopia em solução de Sheather (Teixeira et al., 2011) e o TF-Test Coccidia. A especificidade e a sensibilidade das técnicas de microscopia geralmente são variáveis, estando diretamente ligadas a experiência dos técnicos envolvidos em suas execuções (Teixeira et al., 2011). Embora as técnicas de microscopia sejam mais utilizadas em rotinas laboratoriais, tais técnicas geralmente apresentam menores índices de sensibilidade e especificidade que as técnicas imunológicas (Arrowood \& Sterling, 1989; Teixeira et al., 2011) e moleculares (Meireles et al., 2011; Homem et al., 2012).

Para o diagnóstico imunológico de oocistos presentes em fezes, existem atualmente no mercado kits comerciais de reação imunoenzimática (ELISA) capazes de detectar a presença de coproantígenos do parasito e kits de imunofluorescência direta (IFD) que empregam anticorpos monoclonais antiCryptosporidium marcados com isotiocianato de fluoresceína (Bialek et al., 2002). As técnicas imunológicas, além de apresentarem uma melhor sensibilidade e especificidade na detecção de oocistos em amostras fecais (comparada às técnicas tradicionais de microscopia), podem também reduzir o tempo de trabalho para detecção do parasito (Arrowood \& Sterling, 1989; Garcia \& Shimizu, 1997).

O problema do uso de técnicas imunológicas para detecção de Cryptosporidium spp. em amostras fecais, está no alto custo na aquisição de kits comerciais de Elisa e IFD (Bialek et al., 2002), sendo as técnicas de microscopia mais indicadas para utilização no dia a dia de um laboratório (Weisel et al., 2006). Segundo (Teixeira et al., 2011) a produção de conjugados contendo anticorpos policlonais antiCryptosporidum parvum e isotiocianato de floresceína, pode viabilizar financeiramente o uso da IFD na rotina laboratorial. Além disso, devido a existência de proteínas de superfície comuns entre as diferentes espécies de Cryptosporidium, a utilização do referido conjugado, padronizado com anticorpos policlonais produzidos em coelhos, pode permitir a detecção de oocistos de diferentes espécies do parasito (C. pavum, C. andersoni, C. serpents e C. galli) em amostras fecais de diferentes espécies animais. 
A espécie de Cryptosporidium spp. existente em uma amostra fecal, não pode ser determinada por meio das características morfológicas dos oocistos observadas durante a realização de técnicas de microscopia (Fall et al., 2003), ou ainda, por meio de técnicas imunológicas (Garcia \& Shimizu, 1997), sendo para esse objetivo, sempre necessária a realização de técnicas moleculares (Meireles et al., 2011).

As técnicas moleculares se caracterizam por alta sensibilidade e especificidade no diagnóstico de oocistos de C. parvum em amostras fecais de bovinos (Homem et al., 2012), embora também apresentam um elevado custo para sua realização. A caracterização molecular de Cryptosporidium em bovinos geralmente é realizada por meio da Reação em Cadeia da Polimerase (nested PCR), seguido do sequenciamento dos fragmentos amplificados. O locus mais utilizado é o gene da subunidade $18 \mathrm{~S}$ do RNA ribossômico (18S rRNA), por apresentar cinco cópias por genoma e, pelo fato de caracterizar espécies ou genótipos ainda não classificados (Xiao et al., 2004).

O gene da glicoproteína GP60 vem sendo utilizado na caracterização dos subtipos de $C$. parvum diagnosticados em bovinos (Meireles et al., 2011; Taylan-Ozkan et al., 2016; Baroudi et al., 2017; Matsuura et al., 2017;). Utilizando-se essa glicoproteína, diversos estudos relataram a presença do subtipo IIa de $C$. parvum, que apresenta elevado potencial zoonótico (Baroudi et al., 2017; Matsuura et al., 2017; Meireles et al., 2011; Taylan-Ozkan et al., 2016). Outra ferramenta de biologia molecular utilizada no diagnóstico da criptosporidiose bovina é a PCR em Tempo Real, que apresenta alta sensibilidade e especificidade na detecção de oocistos de $C$. parvum em amostras fecais de bezerros (Homem et al., 2012).

\section{Tratamento e controle}

A ausência de vacinas e drogas eficazes no tratamento de bovinos infectados por Cryptosporidium spp. dificulta diretamente o controle da infecção por estes parasitos, sendo o manejo adequado dos animais, a forma mais indicada para diminuição da criptosporidiose no rebanho (Graaf et al., 1999). Os animais imunocompetentes apresentam sintomatologia clínica durante aproximadamente duas semanas com grande excreção de oocistos, sessando espontaneamente após este período. Para a recuperação clínica dos animais, é essencial o tratamento de suporte, onde a hidratação por meio de fluídoterapia (via parenteral) pode garantir o balanceamento eletrolítico perdido em decorrência da diarreia (Meganck et al., 2014).

Embora a maioria dos autores relate a inexistência de fármacos eficazes no tratamento da criptosporidiose bovina, existem alguns estudos que demonstraram a redução na excreção de oocistos de C. parvum em bezerros tratados com azitromicina, nitazoxanide e paromomycin (Elitok et al., 2005; Nasir et al., 2013). Quando testada em bezerros infectados por C. parvum, a halofuginona apresentou variáveis índices de eficácia (dose dependentes) sobre a redução na excreção de oocistos nas fezes destes animais (Naciri et al., 1993), sendo considerada pelo referido autor uma alternativa no tratamento da criptosporidiose bovina, entretanto, a utilização deste princípio ativo na rotina terapêutica pode ser dificultada devido a sua ausência comercial em vários países.

A administração profilática de colostro hiperimune em bovinos na prevenção da excreção de oocistos de C. parvum em bovinos experimentalmente infectados foi testada em diferentes estudos clínicos, obtendo diferentes índices de eficácia (Fayer et al., 1989; Perryman et al., 1999), não chegando a uma conclusão final sobre o papel da imunoglobulinas maternas na inibição da eliminação de oocistos do parasito por bezerros neonatos.

\section{Considerações finais}

O Cryptosporidium sp. é um dos principais agentes etiológicos causadores de diarreia em bezerros. Quando associada a outros agentes etiológicos (vírus, bactérias ou outros parasitos) esse parasito pode apresentar altos índices de mortalidade em bezerros neonatos. Neste sentido, a ausência de drogas e vacinas eficazes contra estes parasitos, reforça a necessidade do manejo adequado dos animais como a principal forma de controle destes coccídeos em bovinos. 


\section{Referências bibliográficas}

Arrowood, M. J. \& Sterling, C. R. (1989). Comparison of conventional staining methods and monoclonal antibody-based methods for Cryptosporidium oocyst detection. Journal of Clinical Microbiology, 271490-1495.

Baroudi, D., Khelef, D., Hakem, A., Abdelaziz, A., Chen, X., Lysen, C., . . Xiao, L. (2017). Molecular characterization of zoonotic pathogens Cryptosporidium spp., Giardia duodenalis and Enterocytozoon bieneusi in calves in Algeria. Veterinary Parasitology: Regional Studies and Reports, 866-69.

Baxby, D., Blundell, N. \& Hart, C. A. (1984). The development and performance of a simple, sensitive method for the detection of Cryptosporidium oocysts in faeces. The Journal of Hygiene, 92317-323.

Bialek, R., Binder, N., Dietz, K., Joachim, A., Knobloch, J. \& Zelck, U. (2002). Comparison of fluorescence, antigen and PCR assays to detect Cryptosporidium parvum in fecal specimens. Diagnostic Microbiology and Infectious Disease, 43283-288.

Cacciò, S. M., Sannella, A. R., Mariano, V., Valentini, S., Berti, F., Tosini, F. \& Pozio, E. (2013). A rare Cryptosporidium parvum genotype associated with infection of lambs and zoonotic transmission in Italy. Veterinary Parasitology, 191(1-2):128-131.

De Waele, V., Berzano, M., Berkvens, D., Speybroeck, N., Lowery, C., Mulcahy, G. M. \& Murphy, T. M. (2011). Age-stratified Bayesian analysis to estimate sensitivity and specificity of four diagnostic tests for detection of Cryptosporidium oocysts in neonatal calves. Journal of Clinical Microbiology, 49(1):76-84.

Dixon, B., Parrington, L., Cook, A., Pintar, K., Pollari, F., Kelton \& Farber, J. (2011). The potential for zoonotic transmission of Giardia duodenalis and Cryptosporidium spp. from beef and dairy cattle in Ontario, Canada. Veterinary Parasitology, 17520-26.

Elitok, B., Elitok, O. M. \& Pulat, H. (2005). Efficacy of azithromycin dihydrate in treatment of cryptosporidiosis in naturally infected dairy calves. Journal of Veterinary Internal Medicine, 19590593.

Elliot, A., Morgan, U. M. \& Thompsom, R. C. A. (1999). Improved staining method for detecting Cryptosporidium oocysts in stools using malachite green. Journal of General and Applied Microbiology, 45139-142.

Fall, A., Thompson, R. C., Hobbs, R. P. \& Morgan-Ryan, U. (2003). Morphology is not a reliable tool for delineating species within Cryptosporidium. Journal of Parasitology, 89(2):399-402.

Fayer, R. (2004). Sarcocystis spp. in human infections. Clinical Microbiology Reviews, 17(4):894-902.

Fayer, R., Andrews, C., Ungar, B. L. \& B., B. (1989). Efficacy of hyperimmune bovine colostrum for prophylaxis of cryptosporidiosis in neonatal calves. Journal of Parasitology, 75(3):393-397.

Fayer, R., Santín, M. \& Trout, J. M. (2007). Prevalence of Cryptosporidium species and genotypes in mature dairy cattle on farms in eastern United States compared with younger cattle from the same locations. Veterinary Parasitology, 145260-266.

Fayer, R., Santín, M. \& Trout, J. M. (2008). Cryptosporidium ryanae n. sp.(Apicomplexa: Cryptosporidiidae) in cattle (Bos taurus). Veterinary Parasitology, 156(3-4):191-198.

Feitosa, F. L. F., Shimamura, G. M., Roberto, T., Mendes, L. C. N., Peiró, J. R., Féres, F. C., . . . Meireles, M. V. (2008). Importância de Cryptosporidium spp. como causa de diarréia em bezerros. Pesquisa Veterinária Brasileira, 28452-456.

Garcia, L. S. \& Shimizu, R. Y. (1997). Evaluation of nine immunoassay Kits (enzyme immune assay and direct fluorescence) for detection of Giardia lamblia and Cryptosporidium parvum in human fecal specimens. Journal of Clinical Microbiology, 47209-212.

Garro, C. J., Morici, G. E., Utgés, M. E., Tomazic, M. L. \& Schnittger, L. (2016). Prevalence and risk factors for shedding of Cryptosporidium spp. oocysts in dairy calves of Buenos Aires Province, Argentina. Parasite Epidemiology and Control, 1(2):36-41. 
Graaf, D. C., Vanopdenbosch, E., Ortega-Mora, L. M., Abbassi, H. \& Peeters, J. E. (1999). A review of the importance of cryptosporidiosis in farm animals. International Journal for Parasitology, 2912691287.

Henriksen, S. A. \& Pohlenz, J. F. L. (1981). Staining of cryptosporidia by a modified Ziehl-Neelsen technique. Acta Veterinaria Scandinavica, 22594-596.

Homem, C. G., Nakamura, A. A., Silva, D. C., Teixeira, W. F. P., Coelho, W. M. D. \& Meireles, M. V. (2012). Real-time PCR assay targeting the actin gene for the detection of Cryptosporidium parvum in calf fecal samples. Parasitology Research, 1101741-1745.

Hussain, G., Roychoudhury, S., Singha, B. \& Paul, J. (2017). Incidence of Cryptosporidium andersoni in diarrheal patients from southern Assam, India: a molecular approach. European Journal of Clinical and Microbiology \& Infection Diseases, 36(6):1023-1032.

Lindsay, D. S., Upton, S. J., Owens, D. S., Morgan, U. M., Mead, J. R. \& Blagburn, B. L. (2000). Cryptosporidium andersoni $n$. sp. (Apicomplexa: Cryptosporiidae) from cattle, Bos taurus. The Journal of Eukaryotic Microbiology, 4791-95.

Ma, P. \& Soave, R. (1983). Three-step stool examination for cryptosporidiosis in 10 homosexual men with protracted watery diarrhea. Journal of Infectious Diseases, 147824-828.

Matsuura, Y., Matsubayashi, M., Nukata, S., Shibahara, T., Ayukawa, O., Kondo, Y., . . Tani, H. (2017). Report of fatal mixed infection with Cryptosporidium parvum and Giardia intestinalis in neonatal calves. Acta Parasitologica, 62(1):214-220.

Meganck, V., Hoflack, G. \& Opsomer, G. (2014). Advances in prevention and therapy of neonatal dairy calf diarrhoea: a systematical review with emphasis on colostrum management and fluid therapy. Acta Veterinaria Scandinavica, 56(75):1-8. doi: https://doi.org/10.1186/s13028-014-0075-x.

Meireles, M. V., Oliveira, F. P., Teixeira, W. F. P., Coelho, W. M. D. \& Mendes, L. C. N. (2011). Molecular characterization of Cryptosporidium spp. in dairy calves from the state of São Paulo, Brazil. Parasitology Research, 109(3):949-951.

Morgan, U. M., Xiao, L., Monis, P., Sulaiman, I., Pavlasek, I., Blagburn, B., . . Thompson, R. C. A. (2000). Molecular and phylogenetic analysis of Cryptosporidium muris from various hosts. Parasitology, 120(457-464).

Morse, T. D., Nichols, R. A. B., Grimason, A. M., Campbell, B. M., Tembo, K. C. \& Smith, H. V. (2007). Incidence of cryptosporidiosis species in paediatric patients in Malawi. Epidemiology and Infection, 135(1307-1315).

Muhid, A., Robertson, I., Ng, J. \& Ryan, U. (2011). Prevalence of and management factors contributing to Cryptosporidium sp. infection in pre-weaned and post-weaned calves in Johor, Malaysia. Experimental Parasitology, 127(2):534-538.

Naciri, M., Mancassola, R., Yvoré, P. \& Peeters, J. E. (1993). The effect of halofuginone lactate on experimental Cryptosporidium parvum infections in calves. Veterinary parasitology, 45(3):1992017.

Nasir, A., Avais, M., Khan, M. S., Khan, J. A., Hameed, S. \& Reichel, M. P. (2013). Treating Cryptosporidium parvum infection in calves. Journal of Parasitology, 99715-717.

Noordeen, F., Rajapakse, R. P. V. J., Horadagoda, N. U., Abdul-Careem, M. F. \& Arulkanthan, A. (2012). Cryptosporidium, an important enteric pathogen in goats - A review. Small Ruminant Research, 106(2-3):77-82.

Panciera, R. J., Thomassen, R. W. \& Garner, F. M. (1971). Cryptosporidial infection in a calf. Veterinary Pathology, 8(5-6):479-484.

Perryman, L. E., Kapil, S. J., Jones, M. L. \& Hunt, E. L. (1999). Protection of calves against cryptosporidiosis with immune bovine colostrum induced by a Cryptosporidium parvum recombinant protein. Vaccine, 17(2142-2149).

Santín, M., Trout, J. M., Livestock. In: Fayer, R., Xiao, L. (2007). Cryptosporidium and cryptosporidiosis. Florida: CRC Press. Cap. 18, p. 451-484. 
Taylan-Ozkan, A., Yasa-Duru, S., Usluca, S., Lysen, C., Ye, J., Roellig, D. M., . . Xiao, L. (2016). Cryptosporidium species and Cryptosporidium parvum subtypes in dairy calves and goat kids reared under traditional farming systems in Turkey. Experimental Parasitology, 17016-20.

Teixeira, W. F. P., Coelho, W. M. D., Nunes, C. M. \& Meireles, M. V. (2011). Detection of Cryptosporidium parvum oocysts in calf fecal samples by direct immunofluorescence assay. Revista Brasileira de Parasitologia Veterinária, 20(4):269-273.

Wegayehu, T., Karim, R., Anberber, M., Adamu, H., Erko, B., Zhang, L. \& Tilahun, G. (2016). Prevalence and genetic characterization of Cryptosporidium species in dairy calves in Central Ethiopia. PloS One, 11(5): 0154647.

Weisel, T., Dittrich, S., Möhl, I., Adusu, E. \& Jelinek, T. (2006). Evaluation of seven commercial antigen detection tests for Giardia and Cryptosporidium in stool samples. Clinical Microbiology and Infection, 12656-659.

Xiao, L., Fayer, R., Ryan, U. \& Upton, S. J. (2004). Cryptosporidium taxonomy: recent advances and implications for public health. Clinical Microbiological Reviews, 1792-97.

Yap, N. J., Koehler, A. V., Ebner, J., Tan, T. K., Lim, Y. A. L. \& Gasser, R. B. (2016). Molecular analysis of Cryptosporidium from cattle from five states of Peninsular Malaysia. Molecular and Cellular Probes, 30(1):39-43.

Recebido: 28 de maio, 2019.

Aprovado: 29 de junho, 2019.

Publicado: 30 de julho, 2019.

Licenciamento: Este artigo é publicado na modalidade Acesso Aberto sob a licença Creative Commons Atribuição 4.0 (CC-BY 4.0), a qual permite uso irrestrito, distribuição, reprodução em qualquer meio, desde que o autor e a fonte sejam devidamente creditados. 